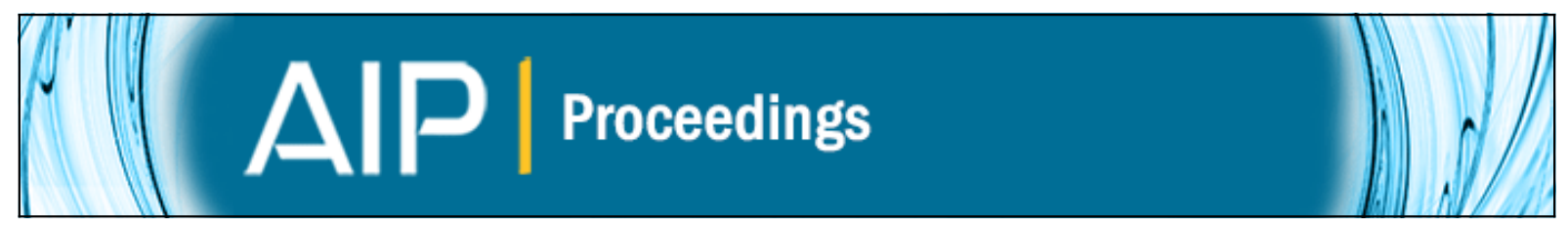

\title{
The effect of roughness on bottom loss from elastic ocean bottoms
}

Marcia J. Isakson and Nicholas P. Chotiros

Citation: AIP Conference Proceedings 1495, 516 (2012); doi: 10.1063/1.4765950

View online: http://dx.doi.org/10.1063/1.4765950

View Table of Contents: http://scitation.aip.org/content/aip/proceeding/aipcp/1495?ver=pdfcov

Published by the AIP Publishing

Articles you may be interested in

The effects of roughness on transmission loss in regions with elastic bottoms

J. Acoust. Soc. Am. 131, 3356 (2012); 10.1121/1.4708580

Backscattering From Scale Models of Elastic Ocean Bottoms with Power-law Roughness

J. Acoust. Soc. Am. 123, 3601 (2008); 10.1121/1.2934769

Rough surface scattering from an elastic scale model of an ocean bottom

J. Acoust. Soc. Am. 114, 2311 (2003); 10.1121/1.4780929

Calculations of acoustic scattering from an elastic ocean bottom

J. Acoust. Soc. Am. 104, 1809 (1998); 10.1121/1.423605

The effect of roughness on acoustic penetration of the ocean bottom

J. Acoust. Soc. Am. 96, 3265 (1994); 10.1121/1.410990 


\title{
The Effect of Roughness on Bottom Loss from Elastic Ocean Bottoms
}

\author{
Marcia J. Isakson ${ }^{\mathrm{a}}$ and Nicholas P. Chotiros ${ }^{\mathrm{a}}$ \\ ${ }^{a}$ Applied Research Laboratories, The University of Texas at Austin, 10000 Burnet Road, Austin Tx, \\ 78758; misakson@arlut.utexas.edu
}

\begin{abstract}
Acoustic interaction with the ocean bottom profoundly affects propagation in shallow waters. However, most forward ocean bottom interactions are modeled as if the bottom were a flat interface or use a simple model to quantify the additional loss. These assumptions either neglect or over-estimate the enhancement of ocean bottom loss due to scattering into the bottom. Scattering from and into elastic bottoms is particularly interesting since it can induce the production of an interface wave. In this study, finite element analysis is used to calculate acoustic scattering from elastic ocean bottoms with varying degrees of roughness. The forward scattering loss from these bottoms is calculated as a function of angle and then compared with the flat bottom reflection coefficient in order to gain insight on the conditions under which enhancement of bottom loss by rough interface scattering is significant.
\end{abstract}

Keywords: Acoustic scattering; sediment acoustics

PACS: 43.30.Hw

\section{INTRODUCTION}

There are many high quality models for propagation and reverberation in shallow water waveguides including parabolic equations, normal and coupled modes and ray theory. However, until recently, ocean propagation modeling has neglected rough interface scattering at low frequency or included it only statistically with models such as the Eckart model.[1] Although finite element modeling has been a long standing technique, only recently has it been applied to ocean modeling due to the large computational resources necessary. In this study, finite elements (FE) will be used to determine the effect of rough interface scattering on bottom loss from an elastic ocean bottom.

\section{MODELING}

The basic model for this work is finite elements, the theory of which is discussed in Ref. 2. The bottom loss model is a mixed finite element/boundary element (FE/BE) model in which the pressure and its normal derivative are calculated on a surface and the pressure at the specular angle is determined through calculation of the Helmholtz/Kirchhoff integral. A typical model is shown in Fig. 1. Here a tapered Gaussian plane wave is incident on the surface to produce the shown scattered 
pressure. The incident wave is calculated using Eq. 11 from Ref. 3. The surface is finely sampled and a pressure $100 \mathrm{~m}$ it at the specular angle is calculated using the Helmholtz/Kirchhoff integral. Verification of the method was performed by comparing a flat interface with an analytic calculation of the reflection coefficient shown in Fig. 2. The two models vary by less the $0.01 \mathrm{~dB}$.

The domain of the FE model is bounded by perfectly matched layers as described in Ref. 4. Realizations of the rough interface are produced using the method described in Ref. 3 using the roughness profile from Ref. 5 scaled to provide the given RMS roughnesses in Fig. 2. The roughness profile used was experimentally measured during the Experimental Validation of Acoustic Measurement Techniques in 2006 off the coast of Isola d'Elba, Italy with a laser line scan system. It can be described as a modified power spectrum in the form:

$$
W(K)=\frac{w_{1}}{\left(K_{L}^{2}+K^{2}\right)^{\gamma / 2}}
$$

Here $K$ is the spatial wavenumber, $K_{L}$ is the wavenumber cutoff, $\gamma$ is the interface roughness slope and $w_{1}$ is the spectral strength which was varied to produce the different values of RMS roughness. The parameters of the roughness, as well as the physical parameters of the water and ocean bottom are given in Tab. 1.

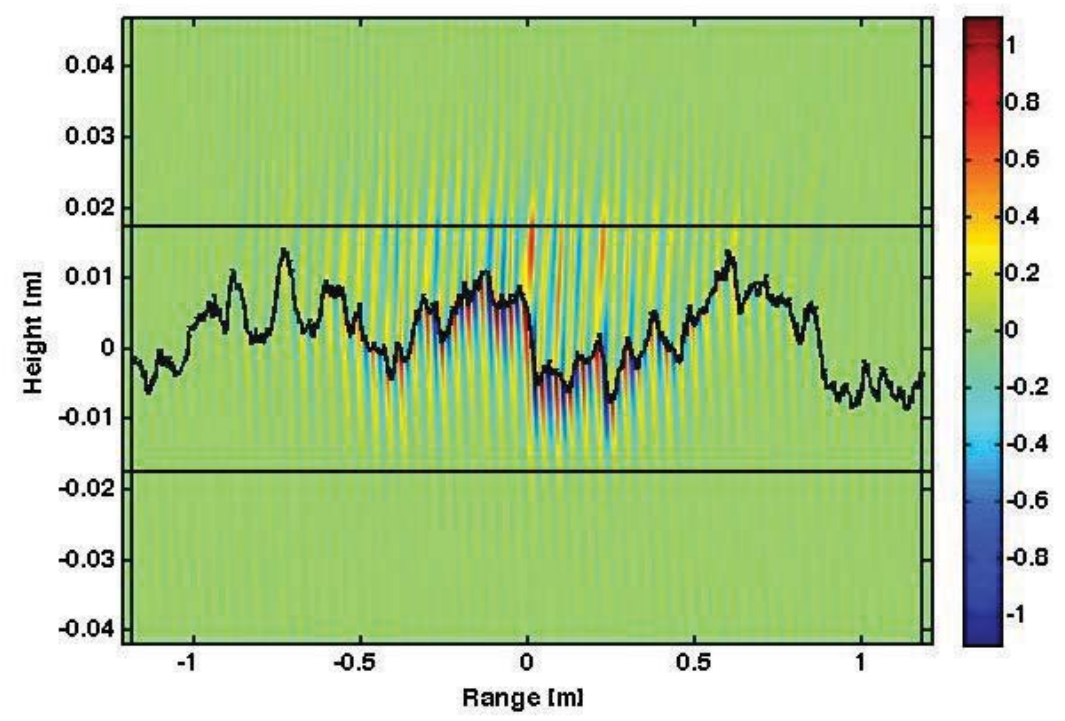

FIGURE 1. Typical domain for finite element scattering model. Shown is the pressure in arbitrary units. The domain is surrounded by perfectly matched layers. [Color online.] 


\begin{tabular}{lc}
\hline \multicolumn{1}{c}{ TABLE 1. Parameter Values for bottom loss calculation. } \\
\hline \multicolumn{1}{c}{ Parameter } & Value \\
\hline Water Sound Speed & $1529 \mathrm{~m} / \mathrm{s}$ \\
Water Attenuation & $0 \mathrm{~dB} / \mathrm{m}$ \\
Water Density & $1026 \mathrm{~kg} / \mathrm{m}^{3}$ \\
Bottom Compressional Sound Speed & $2426 \mathrm{~m} / \mathrm{s}$ \\
Bottom Compressional Attenuation & $0.15 \mathrm{~dB} / \mathrm{m}$ \\
Bottom Shear Speed & $1213 \mathrm{~m} / \mathrm{s}$ \\
Bottom Shear Attenuation & $0.3 \mathrm{~dB} / \mathrm{m}$ \\
Bottom Density & $2400 \mathrm{~kg} / \mathrm{m}^{3}$ \\
Interface Roughness Slope, $\gamma$ & 3.15 \\
Wavenumber Cutoff, $K_{L}$ & $0.215 \mathrm{~cm}^{-1}$ \\
Acoustic Frequency & $10 \mathrm{kHz}$ \\
\hline
\end{tabular}

\section{BOTTOM LOSS FOR ROUGH INTERFACES}

The bottom loss for various values of RMS roughness as compared to a wavelength is shown in Fig. 2. Note the large reflection loss near the intromission angle from the shear mode at 21 degrees grazing as well as the critical angle of the compressional mode at 51 degrees grazing in the flat surface reflection loss curve.

There are two major effects of interface roughness. First, as the roughness increases, the effect of intromission angle from the shear wave is reduced by up to 25 $\mathrm{dB}$ for the highest roughness modeled. On rough surfaces, the local angle encountered by the acoustic wave is changed by the slope of the roughness so a major portion of the wave does not encounter the bottom at the intromission angle. Also, an interface wave is generated due to the changes in the local slope. Although this wave does not propagate far into the water, it produces a pressure at the surface which is included in the Helmholtz/Kirchhoff integral and has the effect of decreasing the bottom loss. Second, as roughness increases, bottom loss near normal incidence also increases as more energy is scattered into the ocean bottom.

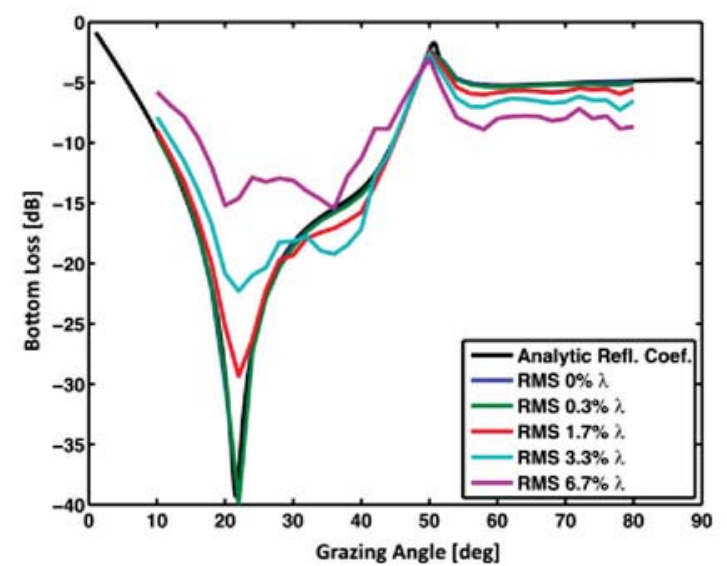

FIGURE 2. Bottom loss for various values of RMS roughness compared to an acoustic wavelength. The black curve is produced using an analytic model while the colored curves are produced using the mixed FE/BE approach. [Color online.] 
In order to observe these effects, the magnitude of the scattered particle velocity field at two angles for a variety of roughness conditions is shown in Fig. 3 and Fig. 4. The incident field has not been included. The particle velocity is shown instead of the familiar pressure since it is the natural unit for elastic solids. In. Fig. 3, the field is shown for a grazing angle of 21 degrees at RMS roughnesses of $0 \%$ of a wavelength (flat), $1.7 \%$ of a wavelength, and $6.7 \%$ of a wavelength. Note that the depth scaling is vastly different from the range scaling so that although the grazing angle is low, the acoustic wave appears to be scattering normally. The incident wave is coming from the left while the scattered wave goes toward the positive range values. In the flat case, the intromission angle is quite pronounced and most of the energy is in the lower layer. Also, the energy is localized within the beam waist of the incident beam. However, for the rougher cases, there is significant energy in the upper layer especially in areas where the local grazing varies greatly from the nominal grazing angle. Also, there is significant energy in areas to the right (positive ranges) indicating the formation of a interface wave in the direction of the incident horizontal wavenumber. This is especially apparent in the case in Fig. $3 \mathrm{~b}$.

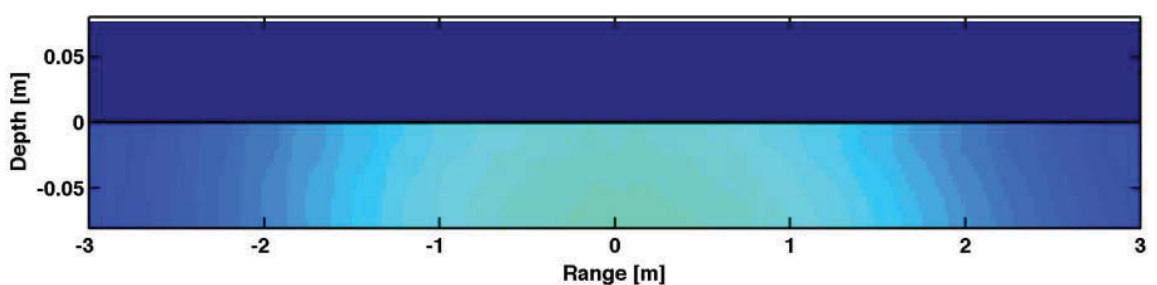

(a)

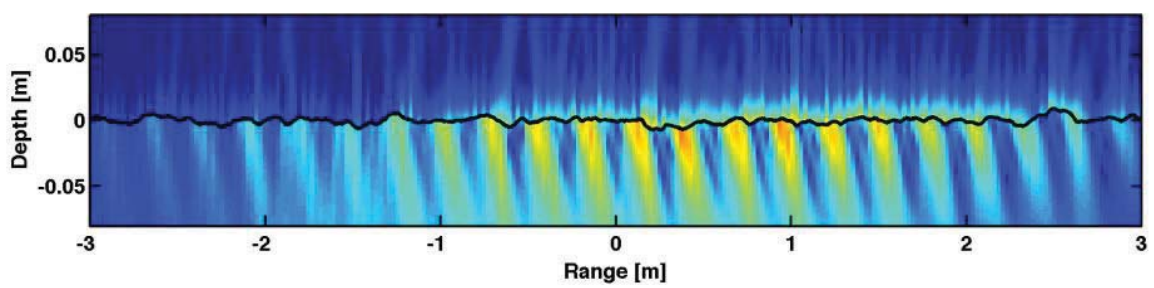

(b)

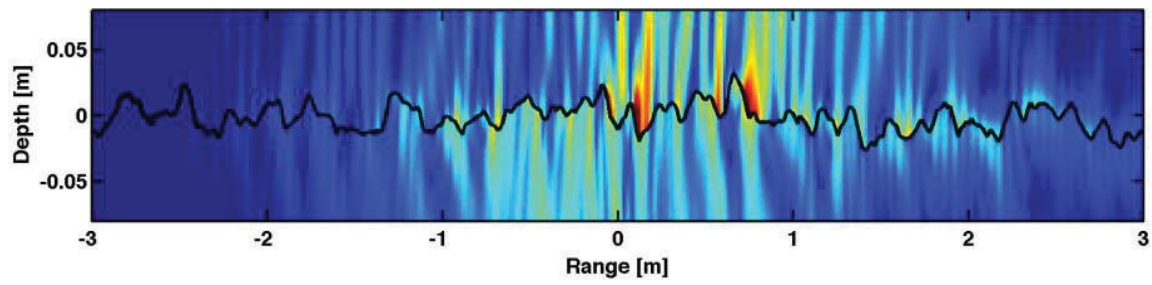

(c)

FIGURE 3. The magnitude of the particle velocity of the scattered wave for an incident wave at 21 degrees grazing. Three cases are shown: (a) RMS roughness $=0 \%$ of an acoustic wavelength, (b) $1.7 \%$ of acoustic wavelength, and (c) $6.7 \%$ of an acoustic wavelength.

In Fig. 4 is shown the magnitude of the particle velocity for three cases at a grazing angle of 70 degrees for the same roughness realizations as Fig. 3. Note that for the flat case, there is significant acoustic energy in the water. However, as the surface is roughened, the local grazing angles vary significantly from the nominal angle 
allowing more energy to be coupled into the bottom. Also, there are local focus areas. Again, there is some evidence of interface wave production, however, at these greater angles, it is not as pronounced since the horizontal component of the incident wavenumber is much less.

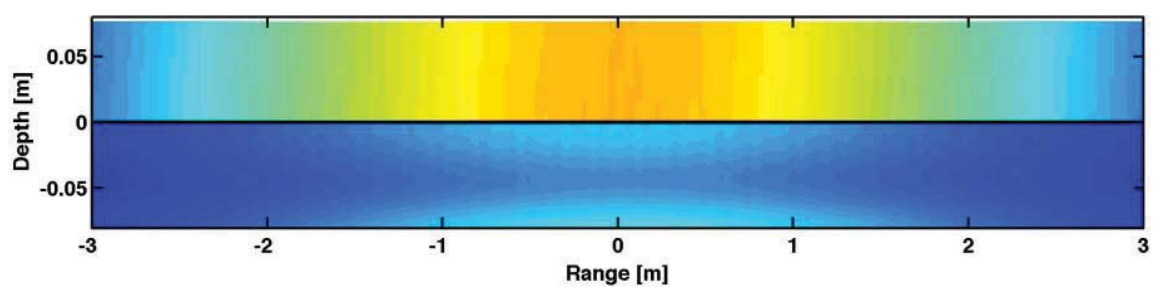

(a)

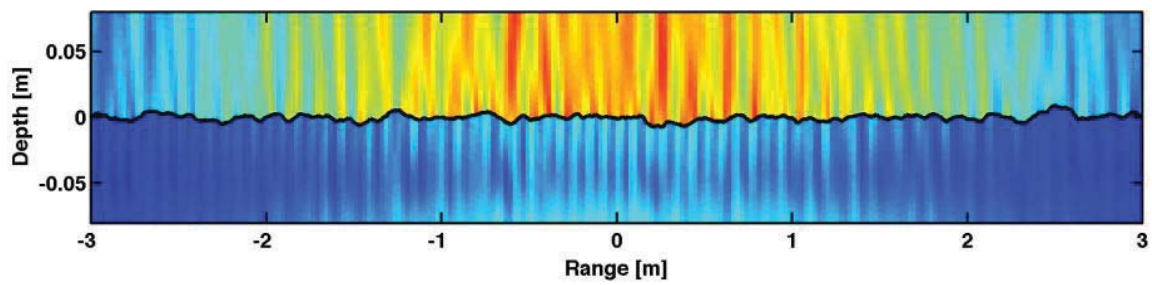

(b)

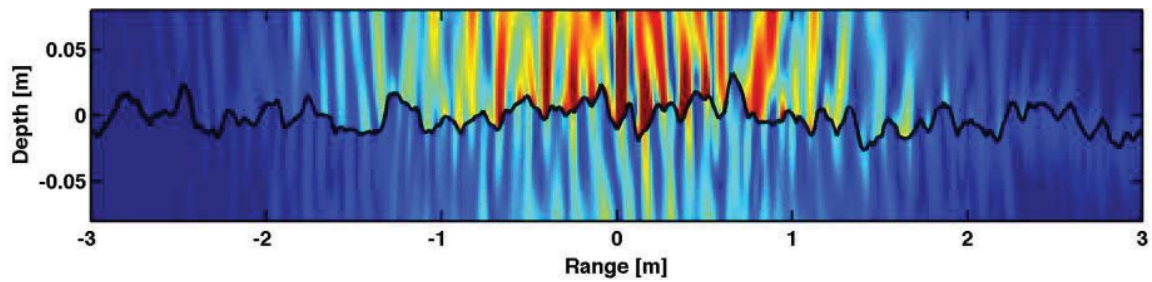

(c)

FIGURE 4. The magnitude of the particle velocity of the scattered wave for an incident wave at 70 deg grazing. Three cases are shown: (a) RMS roughness $=0 \%$ of an acoustic wavelength, (b) $1.7 \%$ of acoustic wavelength, and (c) $6.7 \%$ of an acoustic wavelength. [Color online.]

\section{CONCLUSION}

Seafloor roughness has a significant effect on bottom loss for elastic bottoms even for moderate values of RMS roughness from 1-6\% of an acoustic wavelength. As the roughness increases, the effect of intromission angle of the shear wave is reduced due to changes in local angle. At shallow grazing angles, interface waves are excited for rough interfaces due to the increase of the local grazing angle. At near normal incidence, bottom loss in increased as energy is scattered in to the bottom.

The results of this work indicate that even moderate amounts of interface roughness must be considered when predicting acoustic propagation in shallow water environments as the value of bottom loss is perturbed by up to $25 \mathrm{~dB}$ at shallow grazing angles.

\section{ACKNOWLEDGMENTS}

This work is supported by the Office of Naval Research, Ocean Acoustics. 


\section{REFERENCES}

1. C. Eckart. The scattering of sound from the sea surface. J. Acoust. Soc. Am., 25:560-570, 1953.

2. J. Reddy. An Introduction to the Finite Element Model. McGraw-Hill, New York, N.Y., 2006.

3. E. Thorsos. The validity of the Kirchoff approximation for rough surface scattering using a Gaussian roughness spectrum. J. Acoust. Soc. Am., 83(1):78-92, 1988.

4. J. Berenger. A perfectly matched layer for the adsorption of electromagnetic waves. J. of Comp. Phys., 114:185-200, 1994.

5. M. Isakson, N. Chotiros, and J. N. Piper. Bottom loss measurements in a spatially variable environment at the sediment acoustics experiment 2004. J. Acoust. Soc. Am., In Press, 2012. 